\title{
Assessment of effectiveness of hand hygiene practices amongst food handlers of tertiary care hospital
}

\author{
Jinal Dama ${ }^{1}$, Rajesh Ramteke ${ }^{2, *}$, Navinchandra M. Kaore ${ }^{3}$, Surender Kaur ${ }^{4}$, Shantaram K. Gautam ${ }^{5}$ \\ ${ }^{1} 2^{\text {nd }}$ Professional MBBS Student, ${ }^{2}$ Assistant Professor, ${ }^{3}$ Professor and Head,,${ }^{4,5}$ Assistant Professor, ${ }^{2}$ Dept. of FMT, ${ }^{3-5}$ Dept. of \\ Microbiology, Raipur Institute of Medical Sciences, Raipur, Chhattisgarh, India
}

*Corresponding Author: Rajesh Ramteke

Email: rajeshramteke558@gmail.com

\begin{abstract}
Diseases spread through food still remain a common and persistent problems resulting in appreciable morbidity and occasional mortality. Food handlers may constitute a reservoir of virulent strains and may be vehicles of their transmission to food. Approximately 10 to $20 \%$ of food-borne disease outbreaks are due to contamination by the food handler. In hospitals regular training about food safety and hand hygiene practices for food handlers are done.

So this study was planned to look for efficacy of hand hygiene practices by food handlers against contamination of hand by pathogenic organisms or parasites.

This cross-sectional prospective analytical study was carried out from $1^{\text {st }}$ July to $31^{\text {st }}$ August 2018 in the department of Microbiology, attached to Raipur Institute of Medical Sciences, Raipur attached to tertiary care hospital after due approval of the Institutional Ethics Committee. A convenient sample size of 35 food handlers consenting to the study were included and swabs were collected from the hands of the food handlers just after their procedural hand wash and also the scrapings from the nail bed for fungi \& parasitic forms. Data was maintained in Microsoft Excel and was analyzed using test of proportions.

A total 31/35 (88.5\%) samples taken showed growth with 51 clinical isolates. Beside commensal flora 10 strains of S. aureus and 5 Gram negative isolates like E.coli, Klebsiella and Pseudomonas were isolated by standard microbiological procedures. Two strains each of MRSA and Clindamycin induced Erythromycin resistance were identified whereas one strain of Klebsiella pneumoniae was found to be ESBL. No parasites or fungal elements were detected in the nail scrapping of the study population.

The regular training and health education to maintain the personal hygiene and hand hygiene is a need of the hour to prevent transmission of the pathogenic microorganisms form food handler to Health care professional and/or patients.
\end{abstract}

Keywords: Bacterial contamination, Food borne infections, Health education \& training.

\section{Introduction}

Food handlers especially in a tertiary care hospital with a poor personal hygiene and poor hand hygiene practices can be potential source of infections due to pathogenic microorganisms. Diarrheal diseases are mostly caused by the food-borne and water borne pathogenic microorganisms and amount to 1.9 million deaths globally. ${ }^{1}$

Food handlers who prepares or dispense the food often serves a link between the transmission of the causative pathogen if proper personal hygiene and proper hand washing is not followed. Food handlers may harbor and excrete pathogenic bacteria which may contaminate food they handle from their feces via their fingers, infecting healthy individuals. ${ }^{2,3}$

Compared to other parts of the hand, the area beneath fingernails can be the vector to spread harmful microorganisms through cross contamination. ${ }^{4}$ Human hands are in regular contact with the surrounding environment and a variety of pathogens can reach the mucous membranes in the mouth, nose, eyes and genitals of human being through the hands and consequently contribute to foodborne outbreak. ${ }^{5}$

The illnesses are caused either by the microorganisms themselves or by the toxins they release. ${ }^{6}$ The consumption of foods contaminated by foodborne pathogenic microorganisms and toxins produced by them cause deaths, illnesses, hospitalization, and economic losses. Due to their widespread nature, foodborne diseases, in particular gastro- intestinal infections, represent a very large group of pathologies with a strong negative impact on public health. ${ }^{7}$

According to the National Food Safety Standards in India, the food handlers need to undergo complete check ups annually and are trained repeatedly to maintain the hand hygiene practices while preparing and dispensing the food. Hand washing is a fundamental preventive measure to help protect against the spread of diseases and to reduce the transfer of bacteria from person to person or contaminating the food. ${ }^{8}$ Hands contamination of food handlers can be taken as an indicator of their overall behavior regarding food-related practice and personal hygiene.

Thus, this study was planned to assess the effectiveness the hand hygiene practices employed in terms of contamination of their hands by potential bacterial and parasitic pathogens and to ascertain their burden in food handlers.

\section{Materials and Methods}

This cross-sectional Prospective Analytical study was carried out from $1^{\text {st }}$ July to $31^{\text {st }}$ August 2018 in the department of Microbiology, attached to Raipur Institute of Medical Sciences, Raipur attached to tertiary care hospital after due approval of the Institutional Ethics Committee (IEC). [Approval Letter no. RIMS/ADMIN/DIS/08/04052018 Dated 04.05.2018 from IEC-RIMS Registration No. ECR/969/Inst./CG/2017]. 
A convenient sample size of 35 food handlers of mature age and any gender working in the kitchen or dispensing of food in the tertiary care hospital willing and consenting to the study were included in the study whereas those not consenting were excluded.

\section{Sample Collections \& Processing}

After informed consent from the participants, one swab was collected from the hands of the food handlers just after their procedural hand wash and before any contact with the food or food related items using sterile cotton-tipped swab moistened in the peptone water and placed into a sterile test tube.

Scrapping and material from just underneath the nails was collected in a test tube which was processed by adding normal saline and preparing the saline and iodine mount for presence of parasitic ova, cysts or larvae by microscopy.

All the samples were immediately transported to the bacteriology laboratory of Microbiology department and inoculated over Blood agar (BA) and MacConkeys Agar (MA) plates and was later utilized for the preparation of Direct Gram Stain. The Direct Gram stain results was compared with the isolated colonies on the BA \& MA plates and colonies were identified with the standard microbiological techniques. The identified strain was further processed for Antimicrobial Susceptibility Testing (AST).$^{9,10}$

Detection of Methicillin Resistant Staphylococcus aureus (MRSA) using Cefoxitin disc $30 \mu \mathrm{g}$ and ESBL producing organisms by Phenotypic confirmatory method (Ceftazidime $30 \mu \mathrm{g}$ and Cefdazidime + Clavulanic acid disc $30 / 10 \mu \mathrm{g}$ ) were carried out as per Clinical Laboratory Standard Institute (CLSI) guidelines. ${ }^{10}$

All data was maintained in Microsoft office Excel and was analyzed using statistical tools like tests of proportion.

\section{Observations and Results}

The study included 35 food handlers. Majority of workers included in study were middle aged, i.e. between 30 to 50 years; predominantly males $23 / 35(65.7 \%$ ) and females $12 / 35(34.2 \%)$. All belonged to lower socioeconomic strata.

Off the 35 hand swabs, growth of microorganisms $>10^{3}$ colony forming unit (CFU) was observed in $31 / 35$ (88.5\%). A total of $24 / 35(68.5 \%)$ samples showed growth of single type of bacteria, 11/35 (31.4\%) samples showed growth of poly-microbial and 04/35(11.4\%) showed No growth.

A total of 51 isolates comprising 33/51(60.78\%) Gram positive cocci, 12/51(23.51\%) Gram Positive Bacilli, $5 / 51(9.80 \%)$ Gram negative and 01/51(1.90\%) yeast like organisms were grown in culture which were identified by standard microbiological techniques. Off the Gram Positive, strains identified were Staphylococcus aureus 10/33(30\%), CONS 11/33(33.3\%), Micrococcus 12/33(39.3\%) amongst Gram positive cocci and Bacillus species 12/31(38.7\%) amongst Gram positive bacilli. (Table 1)

Two strains each of E. coli and Klebsiella pneumoniae and one strain of Pseudomonas aeruginosa was amongst 5 Gram negative isolates. One clinical isolate of Candida albicans 01/51(1.90\%) was also isolated and was the only yeast isolated from food handlers. (Table 1)

Direct microscopy by saline and Iodine mount were used for identifying intestinal parasites from nail scrapings. None of the sample showed any positive finding for parasites but artefacts like pollen, vegetative fibers, and epithelial cells were seen.

All the pathogenic isolates were subjected to the Antimicrobial Susceptibility Testing (AST) by Kirby Brewers Disk Diffusion method. Antimicrobial susceptibility for S. aureus showed $90 \%$ susceptibility for Amoxycillin + Clavulanic acid and $60 \%$ susceptibility for Erythromycin and Clindamicin. Vancomycin was found to be $100 \%$ susceptible. (Graph 1). Of the total isolates of S. aureus, 2/10(20\%) strains were Methicillin Resistant S. aureus (MRSA) and 2/10(20\%) strains were found to be Clindamycin induced erythromycin resistance by D-test.

Table 1: Number and speciation of clinical isolates found on the hands of food handlers.

\begin{tabular}{|l|c|c|c|}
\hline \multicolumn{1}{|c|}{ Isolates } & Identified & Organisms Isolated & Number \\
\hline GPC & 33 & Staphylococcus aureus & 10 \\
& & CONS & 11 \\
& & Micrococcus & 12 \\
\hline GNB & 05 & E. coli & 02 \\
& & Pseudomonas & 01 \\
& & Klebsiella & 02 \\
\hline GPB & 12 & Bacillus species & 12 \\
\hline Yeast like & 01 & Candida albicans & 01 \\
\hline \multicolumn{2}{|r|}{ Total } & 51 \\
\hline
\end{tabular}




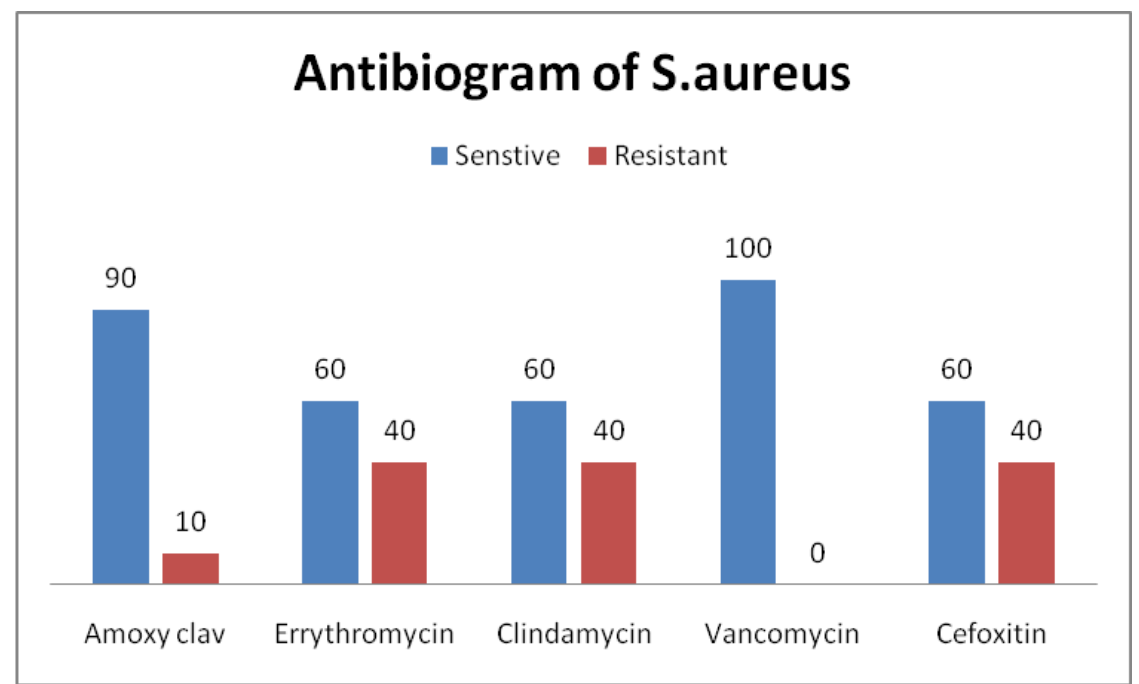

Graph 1: Percentage of sensitive strains of S. aureus to various antibiotics used as per CLSI

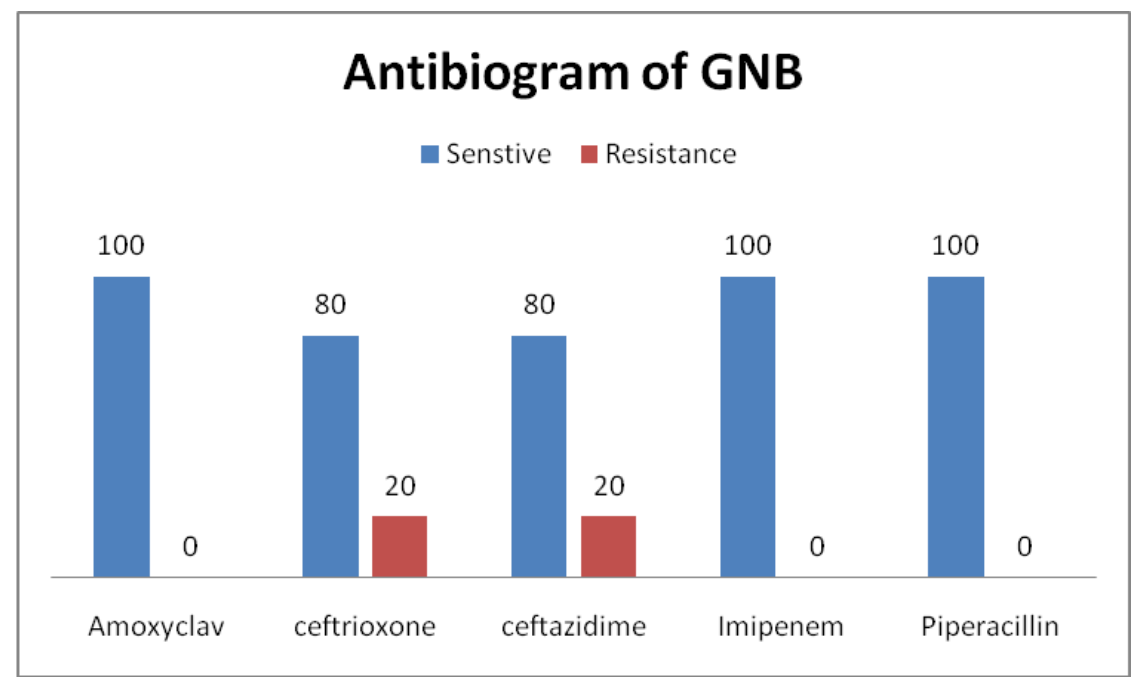

Graph 2: Antibiogram of various gram negative bacilli (GNB) isolated

The AST of all five Gram negative isolates was also determined and is as depicted in Graph 2. Majority of the isolates showed sensitivity above $80 \%$ up to $100 \%$ to primary drugs used. Only one strain of Klebsiella pneumoniae was suspected to be ESBL producer and confirmed by phenotypic confirmation test (PCT) as per guidelines of CLSI and which has also shown resistance to other antimicrobials used as per CLSI.

\section{Discussion}

Food handlers play an important role in transmitting the disease. Any contamination of raw materials, equipment and utensils and/or the hands of food handlers can result in the transmission of intestinal helminthes eggs and bacteria to the consumers or the patients in Heath care settings. ${ }^{11}$

In present study observes similar thing as also by Khurana $\mathrm{S}$ et $\mathrm{al}^{12}$ that, majority of the food handlers belonged to lower socioeconomic strata. It was observed that most of the food handlers didn't follow proper hand hygiene practices after touching dirty materials or after visiting toilet though most of the time soap was not available for hand washing. Even if the soap was available at some places, but they didn't follow proper hand washing practices. Sometimes detergent powder was used or they washed their hands with water only after touching dirty materials, body parts or even after visiting toilets indicates the lack of awareness about food contamination with poor hygienic practices.

In this study the overall contamination level of hands by bacteria was high. This indicated poor personal hygiene practice of the food handlers. The high contamination level in this research was in agreement with the findings of Khurana et al. ${ }^{12}$ The study result shows that about hands of $31 / 35(88.5 \%)$ of the food handlers were contaminated and it was revealed out that this contamination is largely due to the poor personal hygiene practice, lack of environmental sanitation and ignorance of health promotion practice and most importantly hand hygiene practices.

In this study, the participants' hands were contaminated with one or more potentially food borne contaminants and 
had shown 24/35 (68.5\%) mono-microbial and $11 / 35(31.4 \%)$ poly-microbial growth of organisms. Majority of cultures from hands were found to be positive for Gram positive and few Gram negative organisms.

Amongst 33/51(60.78\%) Gram positive cocci, high prevalence of isolation of 12/33(36.36\%) Micrococci and $11 / 33(33.33 \%)$ Coagulase-negative Staphylococci (CONS) was there because they are the normal commensal of the skin followed by Staphylococcus aureus 10/33(30.33\%) are ubiquitously distributed in the environment and strains present in hands and fingers would therefore easily become skin carriers. ${ }^{13}$ Isolation of Staphylococcus aureus indicates poor hand hygiene. Similar results were found by Zagloo et $\mathrm{al}^{14}$ in their study in tertiary care hospital.

Enteric pathogens are among the transient hand flora that can be easily removed by hand washing. Isolation of these organisms includes a feces-to-hand spread and indicating a poor hygiene practices of the food handlers. In the present study amongst Gram negative organisms, different species of Enterobacteriaceae predominantly Klebsiella 2./5 (40\%) and E. coli 2/5 (40\%) were grown supporting the concept of contamination by fecal bacteria due to inadequate hand-washing by the food handlers. ${ }^{15}$ The same type of contaminants was identified by Fawzi et al. ${ }^{16}$ in Alexandria, Egypt (31\% Staphylococcus aureus and $13.8 \%$ Escherichia coli) and Zaglool et al. ${ }^{14}$ in Makkah, Saudi Arabia (17.5\% Staphylococcus aureus). One strain of pseudomonas aeruginosa was isolated in our study which could be from the hospital settings because of the high moisture content over the hands of food handlers. ${ }^{14,3,17}$

Results revealed the role of infected humans in the dissemination and also transmission of MRSA strains to hospital food samples. Among the isolated S. aureus 2/10 (20\%) were MRSA, 2/10 (20\%) were Inducible Clindamycin positive by $\mathrm{D}$ test. Similarly Castro et $\mathrm{al}^{18}$ found role of food handlers in transmission of MRSA strains into the food samples in his study. Since research findings and investigations have suggested that staphylococcal food borne disease is largely due to faulty food handling practices, it is important to give proper training to food handlers in order to prevent the contamination of foods

In our study, ESBL producing Klebsiella were isolated from hand swabs of food handlers which are a cause of concern. Similar results were obtained by Sande et al. ${ }^{19}$

In present study, not a single microscopically identifiable morphological form of any intestinal parasite was detected from scraping samples collected from nail bed of food handler which was similar to observation by Zaglool et $\mathrm{al}^{14}$ but study by Saeed et $\mathrm{al}^{20}$ showed positive finding. This may be as a result of good personal hygiene as well as anti-helminthic treatment routinely administered to all the food handlers as well as continual training for maintaining the hand hygiene.

Similar kind of studies are required regularly to assess the effectiveness of hand hygiene practices in hospital settings as well as in other food industries to know the burden of transient pathogenic organisms responsible for transmission of infections in hospitals and community.

\section{Acknowledgement}

We would like to acknowledge the ICMR for choosing the important topic under short term studentship program. We would like to extend our thanks to Mr. Khomlal Sahu, Technician, Microbiology, for all the technical help in carrying out the study.

\section{Conclusion}

In the present study, bacterial load in food handlers was high with preponderance of pathogenic organism with an encouraging results that no parasitic forms were observed in samples collected from nail beds. Good personal and hand hygienic practices along with hygienic food-handling practices are an effective means for preventing the transmission of pathogens from food-handlers to patients and health care workers. Thus to conclude, regular health checkups, screening of food handlers for potential bacterial pathogens and training for food handling and hand hygiene practices has to be incorporated in hospital setting.

\section{Conflict of Interest: None.}

\section{References}

1. Schlundt J, Toyofuku H, Jansen J. Emerging food-borne zoonoses. Rev Sci Tech 2004;23:513-5.

2. Fendler EJ, Dolan MJ, Williams RA. Hand washing and gloving for food protection: Part I. Examination of the evidence. Dairy Food Environ Sanit 1998;18:814-23.

3. Mohtaram N, Siavash M, Soudeh K, Maryam S \& Masoumeh N. Bacterial assessment of food handlers in Sari City, Mazandaran Province, north of Iran. J Infect Public Health 2017;10:171-6.

4. M. Bas, A.S. Ersun, G. Kivanc. The evaluation of food hygiene knowledge, attitudes and practices of food handlers in food business in Turkey Food Control 2006;17(4):317-22

5. Tan S.L., Lee H.T., Abubakar F., Abdulkarim, M.S., Rukayadi, Y., Mahyudin, N.A. Microbiological quality of food handlers hands at primary school in hulu Langat district Malaysia. Int Food Res J 2013;20(5):2973-7.

6. Centers for Disease control and Prevention (2005) Foodborne Illness. International Food Safety Consultancy.

7. World Health Organization. Food Safety and Foodborne Illness- Fact sheet No.237, 2007

8. Lambrechts AA, Human IS, Doughari JH, Lues JFR. Bacterial contamination of the hands of food handlers as indicator of hand washing efficacy in some convenient food industries. Pak J Med Sci 2014;30(4):755-8.

9. Collee. J, Fraser. A, Marmion. B, Simmons. A. Mackie \& McCartney Practical Medical Microbiology, 14(ed),21, 38798.

10. National Committee for Clinical Laboratory Standards. Performance standards for antimicrobial susceptibility testing ;27 th informational supplement Wayne, Pa:National Committee for Clinical Laboratory Standards;2017(M100S24).

11. 11. Mohan U, Mohan V, Raj K. A Study of Carrier State of S. typhi, Intestinal Parasites \& Personal Hygiene amongst Food Handlers in Amritsar City. Indian J Community Med 2006;31:60-1. 
12. Khurana S, Taneja N, Thapar R, Sharma M, Malla N. Intestinal bacterial and parasitic infections among food handlers in a tertiary care hospital of North India. Trop Gastroenterol 2008.29;4:207-9.

13. Lues JFR, Tonder IV. The occurrence of indicator bacteria on hands and aprons of food handlers in the delicatessen sections of a retail group. Food Control 2007;18:326-32.

14. D. A. Zaglool, Y. A. Khodari, and M. U. Farooq Prevalence of intestinal parasites and bacteria among food handlers in a tertiary care hospital. Niger Med J 2011;52(4):266-70.

15. Andargie G, Kassu A, Moges F, Tiruneh M, Huruy K. Prevalence of bacteria and intestinal parasites among foodhandlers in Gondar town, northwest Ethiopia. J Health Popul Nutr 2008;26:451-5.

16. Fawzi M, Gomaa NF, Bakr WMK., Assessment of hand washing facilities, personal hygiene and the bacteriological quality of hand washes in some grocery and dairy shops in Alexandria, Egypt. J Public Health Assoc 2009; 84(1\&2):7293.

17. Assefa T, Tasew H, Wondafrash B, Beker J. Assessment of Bacterial Hand Contamination and Associated Factors among Food Handlers Working in the Student Cafeterias of Jimma
University Main Campus, Jimma, South West Ethiopia. $J$ Community Med Health Educ 2015'5:345.

18. Castro A, Santos C, Meireles H, Silva J, Teixeira P. Food handlers as potential sources of dissemination of virulent strains ofStaphylococcus aureus in the community. J Infect Public Health 2016;9:153-60.

19. Sande S, Basak S Sande V, Tawade V. Screening of Food Handlers for Intestinal Parasites and Enter opathogenic Bacteria in a Tertiary Care Hospital. Int J Health Sci Res 2014;4(5):88-94.

20. Saeed HA, Hamid HH. Bacteriological and parasitological assessment of food handlers in the Odurman area of Sudan. $J$ Microbiol Immunol Infect 2010;43:70-3.

How to cite this article: Dama J, Ramteke R, Kaore NM, Kaur S, Gautam SK. Assessment of effectiveness of hand hygiene practices amongst food handlers of tertiary care hospital. J Community Health Manag 2019;6(1):25-9. 